\title{
Hermite subdivision on manifolds via parallel transport
}

\author{
Caroline Moosmüller ${ }^{1}$ iD
}

Received: 27 April 2016 / Accepted: 18 January 2017/

Published online: 16 May 2017

(C) The Author(s) 2017. This article is an open access publication

\begin{abstract}
We propose a new adaption of linear Hermite subdivision schemes to the manifold setting. Our construction is intrinsic, as it is based solely on geodesics and on the parallel transport operator of the manifold. The resulting nonlinear Hermite subdivision schemes are analyzed with respect to convergence and $C^{1}$ smoothness. Similar to previous work on manifold-valued subdivision, this analysis is carried out by proving that a so-called proximity condition is fulfilled. This condition allows to conclude convergence and smoothness properties of the manifold-valued scheme from its linear counterpart, provided that the input data are dense enough. Therefore the main part of this paper is concerned with showing that our nonlinear Hermite scheme is "close enough", i.e., in proximity, to the linear scheme it is derived from.
\end{abstract}

Keywords Hermite subdivision $\cdot$ Manifolds subdivision $\cdot C^{1}$ analysis $\cdot$ Proximity

Mathematics Subject Classification (2010) 41A25 - 65D17 · 53A99

\section{Introduction}

Hermite subdivision is an iterative method for constructing a curve together with its derivatives from discrete point-vector data. It has mainly been studied in the linear

Communicated by: Tomas Sauer

The author gratefully acknowledges support by the doctoral program "Discrete Mathematics", funded by the Austrian Science Fund FWF under grant agreement W1230.

Caroline Moosmüller

moosmueller@tugraz.at

1 Institut für Geometrie, TU Graz, Kopernikusgasse 24, 8010 Graz, Austria 
setting, where many results concerning convergence and smoothness are available, such as $[3,4,6-8,14]$ and others.

In a recent paper [15] we propose an analogue of linear Hermite schemes in manifolds which are equipped with an exponential map. This construction works via conversion of vector data to point data, and makes use of the well-established methods of non-Hermite subdivision in manifold, see [9] for an overview. The present paper investigates manifold analogues of Hermite subdivision rules which work directly with vectors and employ the parallel transport operators available in Riemannian manifolds and also in Lie groups. Our motivation for doing subdivision in this way is to use only such operations which are intrinsic to the underlying geometry and which therefore commute with isomorphisms of the respective geometric structures.

The $C^{1}$ convergence analysis of the nonlinear schemes we obtain by the parallel transport approach is provided from their linear counterparts by means of a proximity condition for Hermite schemes introduced by [15]. This condition allows to conclude $C^{1}$ convergence of the manifold-valued scheme if it is "close enough" to a $C^{1}$ convergent linear one. Similar to most previous results on manifold subdivision, $C^{1}$ convergence can only be deduced if the input data are dense enough.

The paper is organized as follows: In Section 2 we recapitulate Hermite subdivision on both linear spaces and manifolds. Section 3 discusses parallel transport and geodesics, which we use in Section 4 to define the parallel transport analogue of a linear Hermite scheme. The main part of this paper is concerned with proving that the proximity condition holds between the parallel transport Hermite scheme and the linear scheme it is derived from (Section 5). The results are then stated in Section 6.

Throughout this paper we use as an instructive example a certain non-interpolatory Hermite scheme which is the de Rham transform [5] of a scheme proposed by [13].

\section{Hermite subdivision: basic concepts}

In this section we recall some known facts about linear Hermite subdivision and introduce a generalized concept of Hermite subdivision for manifold-valued data.

\subsection{Linear hermite subdivision}

The data to be refined by a linear Hermite subdivision scheme consists of a pointvector sequence, where we assume that both point and vector sequence take values in the same finite dimensional vector space $V$. The space of all such point-vector sequences is denoted by $\ell\left(V^{2}\right)$, and an element of this space is written as $\left(\begin{array}{c}p \\ v\end{array}\right)$.

A linear subdivision operator $S_{A}$ is a map $\ell\left(V^{2}\right) \rightarrow \ell\left(V^{2}\right)$, which is defined by

$$
S_{A}\left(\begin{array}{l}
p \\
v
\end{array}\right)_{i}=\sum_{j \in \mathbb{Z}} A_{i-2 j}\left(\begin{array}{c}
p_{j} \\
v_{j}
\end{array}\right), \quad i \in \mathbb{Z}, \quad\left(\begin{array}{c}
p \\
v
\end{array}\right) \in \ell\left(V^{2}\right),
$$

where the finitely supported sequence $A \in \ell\left(L(V)^{2 \times 2}\right)$ is called mask. 
A linear Hermite subdivision scheme is the procedure of constructing $\left(\begin{array}{l}p^{1} \\ v^{1}\end{array}\right)$, $\left(\begin{array}{c}p^{2} \\ v^{2}\end{array}\right), \ldots$ from input data $\left(\begin{array}{c}p^{0} \\ v^{0}\end{array}\right) \in \ell\left(V^{2}\right)$ by the rule

$$
D^{n}\left(\begin{array}{c}
p^{n} \\
v^{n}
\end{array}\right)=S_{A}^{n}\left(\begin{array}{c}
p^{0} \\
v^{0}
\end{array}\right)
$$

where $D \in L(V)^{2 \times 2}$ is the block-diagonal dilation operator

$$
D=\left(\begin{array}{ll}
1 & 0 \\
0 & \frac{1}{2}
\end{array}\right) \text {. }
$$

Here a constant $c$ is to be understood as $c \operatorname{id}_{V}$.

A linear Hermite subdivision operator or scheme is called interpolatory if its mask satisfies $A_{0}=D$ and $A_{2 i}=0$ for all $i \in \mathbb{Z} \backslash 0$.

We always assume a linear Hermite scheme to satisfy the spectral condition, which is a useful assumption for the analysis of linear Hermite schemes [3, 4, 6, 14]. We require that up to a parameter shift the subdivision operator reproduces a degree 1 polynomial and its derivative

$$
\left(\begin{array}{c}
v+i w \\
w
\end{array}\right)_{i \in \mathbb{Z}} \text { for } v, w \in V .
$$

To be precise, we require that there exists $\varphi \in \mathbb{R}$ such that

$$
S_{A}\left(\begin{array}{c}
v+(i+\varphi) w \\
w
\end{array}\right)_{i \in \mathbb{Z}}=\left(\begin{array}{c}
v+\frac{i+\varphi}{2} w \\
\frac{1}{2} w
\end{array}\right)_{i \in \mathbb{Z}},
$$

for all $v, w \in V$. This condition is equivalent to the requirement that there exists $\varphi \in \mathbb{R}$ such that the constant sequence $c_{i}=\left(\begin{array}{l}v \\ 0\end{array}\right)$ and the linear sequence $\ell_{i}=\left(\begin{array}{c}(i+\varphi) v \\ v\end{array}\right)$ for $i \in \mathbb{Z}, v \in V$ respectively obey the rules

$$
S_{A} c=c \quad \text { and } \quad S_{A} \ell=\frac{1}{2} \ell .
$$

The spectral condition can also be expressed by means of the mask $A=\left(\begin{array}{ll}a & b \\ c & d\end{array}\right)$. It is equivalent to

$$
\begin{aligned}
& \sum_{j \in \mathbb{Z}} a_{i-2 j}=1, \quad \sum_{j \in \mathbb{Z}} c_{i-2 j}=0, \\
& \sum_{j \in \mathbb{Z}} a_{i-2 j} j+b_{i-2 j}=\frac{1}{2}(i-\varphi), \quad \sum_{j \in \mathbb{Z}} c_{i-2 j} j+d_{i-2 j}=\frac{1}{2},
\end{aligned}
$$

for all $i \in \mathbb{Z}$ and some $\varphi \in \mathbb{R}$, which indicates the parameter transform. Equation 3 is equivalent to the reproduction of constants, whereas (4) expresses the reproduction of linear functions.

\subsection{Hermite subdivision on manifolds}

We would like to consider Hermite subdivision in the more general setting of manifolds. In this context, tangent vectors serve as point-vector input data for Hermite 
subdivision. Therefore, the input data is sampled from the tangent bundle $T M=$ $\bigcup_{x \in M} T_{x} M$ of a manifold $M$. Its associated sequence space is denoted by $\ell(T M)$. In order to retain the analogy to the linear case, an element of $\ell(T M)$ is written as a pair $\left(\begin{array}{l}p \\ v\end{array}\right)$ consisting of an $M$-valued point sequence $p$ and a vector sequence $v$ which takes values in the appropriate tangent space, i.e., $v_{i} \in T_{p_{i}} M$ for $i \in \mathbb{Z}$.

A subdivision operator $U$ on $T M$ is a map that takes arguments in $\ell(T M)$ and produces again a point-vector sequence. It must satisfy

(i) $L^{2} U=U L$, where $L$ is the left shift operator, and

(ii) $U$ has compact support, that is, there exists $N \in \mathbb{N}$ such that both $U\left(\begin{array}{l}p \\ v\end{array}\right)_{2 i}$ and $U\left(\begin{array}{l}p \\ v\end{array}\right)_{2 i+1}$ only depend on $\left(\begin{array}{c}p_{i-N} \\ v_{i-N}\end{array}\right), \ldots,\left(\begin{array}{l}p_{i+N} \\ v_{i+N}\end{array}\right)$ for all $i \in \mathbb{Z}$ and sequences $\left(\begin{array}{l}p \\ v\end{array}\right)$.

Let $D: \ell(T M) \rightarrow \ell(T M)$ be the dilation operator

$$
\left(\begin{array}{c}
p \\
v
\end{array}\right) \mapsto\left(\begin{array}{c}
p \\
\frac{1}{2} v
\end{array}\right),
$$

which is an analogue of the block-diagonal operator $D$ defined in Section 2.1.

An Hermite subdivision scheme is the procedure of constructing $\left(\begin{array}{l}p^{1} \\ v^{1}\end{array}\right),\left(\begin{array}{c}p^{2} \\ v^{2}\end{array}\right), \ldots$ from input data $\left(\begin{array}{c}p^{0} \\ v^{0}\end{array}\right) \in \ell(T M)$ by the rule

$$
D^{n}\left(\begin{array}{c}
p^{n} \\
v^{n}
\end{array}\right)=U^{n}\left(\begin{array}{c}
p^{0} \\
v^{0}
\end{array}\right)
$$

An Hermite subdivision operator or scheme is called interpolatory if $U\left(\begin{array}{l}p \\ v\end{array}\right)_{2 i}=$ $D\left(\begin{array}{l}p \\ v\end{array}\right)_{i}$ for all $\left(\begin{array}{l}p \\ v\end{array}\right)$ and $i \in \mathbb{Z}$.

Note that these definitions are direct generalizations of the concepts introduced in Section 2.1: Every linear subdivision operator satisfies conditions (i) and (ii) from above. If $U$ is linear then the definition of (interpolatory) Hermite subdivision scheme is equivalent to the one given in Section 2.1.

\section{$2.3 C^{1}$ convergence}

To a sequence $p^{n}$ of points in a vector space we associate a curve $\mathcal{F}_{n}\left(p^{n}\right)$, which is the piecewise linear interpolant of $p^{n}$ on the grid $2^{-n} \mathbb{Z}$.

We say that a point-vector sequence $\left(\begin{array}{c}p^{n} \\ v^{n}\end{array}\right)$ is $C^{1}$ convergent, if $\mathcal{F}_{n}\left(p^{n}\right)$ resp. $\mathcal{F}_{n}\left(v^{n}\right)$ converge uniformly on compact intervals to a continuously differentiable curve resp. its derivative. If the point-vector sequence is manifold-valued, then we require that the above is true in a chart.

A Hermite scheme defined by the subdivision operator $U$ is said to be $C^{1}$ convergent, if the point-vector sequence $\left(\begin{array}{c}p^{n} \\ v^{n}\end{array}\right)$ constructed via $D^{n}\left(\begin{array}{c}p^{n} \\ v^{n}\end{array}\right)=U^{n}\left(\begin{array}{c}p^{0} \\ v^{0}\end{array}\right)$ is $C^{1}$ convergent. 

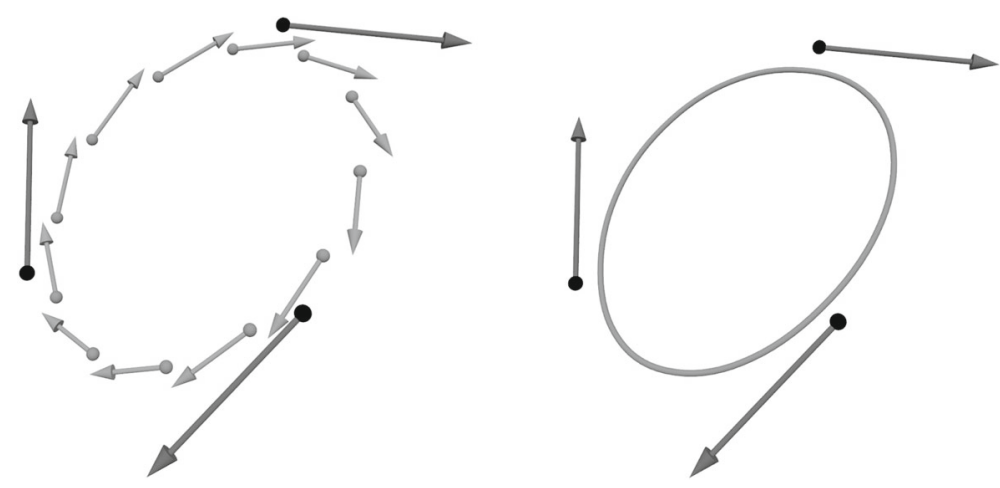

Fig. 1 The linear non-interpolatory Hermite subdivision scheme of Example 1. Left: Input data and second iteration step. Right: Input data and limit curve

Example 1 We consider the de Rham transform [5] of one of the interpolatory linear Hermite schemes introduced by [13], see Fig. 1. It is a non-interpolatory scheme with mask

$$
\begin{aligned}
& A_{-2}=\frac{1}{8}\left(\begin{array}{rr}
\frac{48}{25} & -\frac{29}{25} \\
\frac{29}{50} & \frac{13}{20}
\end{array}\right), \quad A_{-1}=\frac{1}{8}\left(\begin{array}{rr}
\frac{152}{25} & -\frac{31}{25} \\
\frac{29}{50} & \frac{277}{100}
\end{array}\right), \\
& A_{0}=\frac{1}{8}\left(\begin{array}{rr}
\frac{152}{25} & \frac{31}{25} \\
-\frac{29}{50} & \frac{277}{100}
\end{array}\right), \quad A_{1}=\frac{1}{8}\left(\begin{array}{rr}
\frac{48}{25} & \frac{29}{25} \\
-\frac{29}{50} & \frac{13}{20}
\end{array}\right) .
\end{aligned}
$$

In [5] it is shown that the spectral condition is satisfied and that this scheme is $C^{1}$ convergent.

\section{Parallel transport and geodesics}

Using parallel transport and geodesics, we are going to adapt linear Hermite subdivision to work on manifold data. We here discuss these concepts for submanifolds of Euclidean space (i.e., surfaces) and for matrix groups, even though they belong to the more general classes of Riemannian manifolds resp. Lie groups. The reason is that the convergence and smoothness analysis presented in Section 5 can be reduced to the special cases of surfaces and matrix groups.

\subsection{Surfaces}

On a surface $M$ in $\mathbb{R}^{n}$ we consider vector fields $V(t)$ along a curve $g(t)$, i.e., we require that $V(t) \in T_{g(t)} M$ for all $t$. We say that such a vector field $V$ is parallel along $g$ if its derivative is orthogonal to $M$. Equivalently, the projection of $\dot{V}$ to the tangent space $T_{g(t)} M$ vanishes for all $t$, i.e.

$$
\frac{D V}{d t}:=(\dot{V})^{\operatorname{tang}}=0 \text {. }
$$


Therefore, parallel vector fields are the solutions of the linear differential Eq. 5.

Let the curve $g$ connect the points $p$ and $m$ on $M$, i.e., $g(0)=p$ and $g(1)=m$. The parallel transport along $g$, denoted by $\mathrm{P}_{p}^{m}: T_{p} M \rightarrow T_{m} M$, is defined as follows: $\mathrm{P}_{p}^{m}(v)$ means $V(1)$, where $V$ is the parallel vector field along $g$ with initial value $V(0)=v$.

Parallel transport along $g$ satisfies

$$
\mathrm{P}_{q}^{m} \circ \mathrm{P}_{p}^{q}=\mathrm{P}_{p}^{m}
$$

where $q$ is any point on the curve. Furthermore, it is an isometry, that is $\left\|\mathrm{P}_{p}^{m}(v)\right\|=$ $\|v\|$. This is not difficult to show: For two vector fields $V, W$ along $g$ a product rule holds:

$$
\frac{d}{d t}\langle V, W\rangle=\left\langle\frac{D V}{d t}, W\right\rangle+\left\langle V, \frac{D W}{d t}\right\rangle .
$$

If $V$ is parallel along $g$, then Eq. 7 implies that $\frac{d}{d t}\langle V(t), V(t)\rangle=0$, i.e., $\|V(t)\|$ is constant for all $t$. So $\mathrm{P}_{p}^{m}$ is an isometry.

In addition to parallel transport, we need the concept of geodesics. A geodesic is a curve $g$ on $M$ such that $\dot{g}$ is parallel along $g$, i.e., a curve which satisfies the differential equation

$$
\frac{D \dot{g}}{d t}=0 .
$$

It is useful to express geodesics by means of the exponential mapping, which is defined as follows: $\exp _{p}(v)$ means $g(1)$, where $g$ is the geodesic starting at the point $p$ with tangent vector $v$. A geodesic $g$ can then be written as $g(t)=\exp _{p}(t v)$.

We mention that $\frac{D}{d t}$, parallel transport, geodesics and exponential mapping are actually concepts of Riemannian geometry. Here they are described only for the special case of surfaces in Euclidean space. For details we refer to textbooks on differential geometry, e.g. [1].

\subsection{Matrix groups}

This section discusses parallel transport and geodesics in matrix groups, i.e., subgroups of $\mathrm{GL}(n, \mathbb{R})$.

We use the matrix exponential function $\exp (v)=\sum_{k=0}^{\infty} \frac{1}{k !} v^{k}$ to define an exponential mapping by $\exp _{p}(v)=p \exp \left(p^{-1} v\right)$. Then a geodesic ${ }^{1} g$ starting at the point $p$ and tangent vector $v$ is defined by

$$
g(t)=\exp _{p}(t v)
$$

The curve $g(t)$ is a left translate of the 1-parameter subgroup $\exp \left(t p^{-1} v\right)$, and it is also a right translate, since $p \exp \left(p^{-1} v\right)=\exp \left(v p^{-1}\right) p$. We define three different

\footnotetext{
${ }^{1}$ We call these curves geodesics to emphasize the analogy to the Riemannian case. Note that in the group case we define geodesics via the exponential map, but in the Riemannian case, we define the exponential map via geodesics.
} 
parallel transports ${ }^{+} \mathrm{P}_{p}^{m},-\mathrm{P}_{p}^{m}$ and ${ }^{0} \mathrm{P}_{p}^{m}$ on $G$, which are mappings of $T_{p} G$ to $T_{m} G$. The first two are given by left resp. right multiplication, that is

$$
{ }^{+} \mathrm{P}_{p}^{m}(v)=m p^{-1} v \quad \text { and } \quad{ }^{-} \mathrm{P}_{p}^{m}(v)=v p^{-1} m .
$$

Let $g(t)=\exp _{p}(t v)$ be the geodesic connecting $p$ and $m$. Denote by $\mu_{p, m}$ the geodesic midpoint of $p$ and $m$, i.e., $\mu_{p, m}=g\left(\frac{1}{2}\right)$. Then the third kind of parallel transport is defined by

$$
{ }^{0} \mathrm{P}_{p}^{m}(v)=\mu_{p, m} p^{-1} v p^{-1} \mu_{p, m} .
$$

Therefore, as in the Riemannian case, an exponential mapping, geodesics and parallel transport can be defined in matrix groups. ${ }^{2}$

\subsection{Unified notation}

The following sections treat surfaces and matrix groups simultaneously. Therefore we introduce a unified notation.

$M$ means either a surface or a matrix group. The exponential mapping of $M$ is denoted by $\exp _{p}(v)$. In the surface case, $\mathrm{P}_{p}^{m}$ denotes the parallel transport along the geodesic connecting $p$ and $m$. If $M$ is a matrix group, $\mathrm{P}_{p}^{m}$ refers to one of the parallel transports introduced in Section 3.2.

Following [20], we introduce the symbols $\oplus$ and $\ominus$ which are analogues of pointvector addition and difference. For $p, q \in M$ and $v \in T_{p} M$, let

$$
p \oplus v=\exp _{p}(v) \text { and } q \ominus p=\exp _{p}^{-1}(q) .
$$

Note that in the matrix group case the $\oplus$ and $\ominus$ operations are invariant w.r.t. both left and right multiplication.

While $\oplus$ is always smooth and often globally defined (this is the case in both matrix groups and complete surfaces $[11,16]), \ominus$ is in general only smooth in some neighborhood of $p$. Our results in Section 5 are based on [15], which only considers "dense enough" input data. We therefore assume that $\ominus$ is always smooth. As in the matrix group case, we define the midpoint of two points $p, q$ on $M$ : If $g$ is the geodesic connecting $p$ and $q$, then

$$
\mu_{p, q}=g\left(\frac{1}{2}\right)=p \oplus \frac{1}{2}(q \ominus p) .
$$

\footnotetext{
${ }^{2}$ In fact a more general statement is true, which also gives a connection to the case of Riemannian manifolds: On Lie groups, three operators $+\frac{D}{d t},-\frac{D}{d t}$ and ${ }^{0} \frac{D}{d t}$ can be defined, which map a vector field along a curve to another vector field along the same curve. They all define the same geodesics, namely (8) and induce the three parallel transports from above. While ${ }^{+} \mathrm{P}_{p}^{m}$ and ${ }^{-} \mathrm{P}_{p}^{m}$ are independent of the curve connecting $p$ and $m$, Definition (9) is only valid if the curve under consideration is the geodesic connecting $p$ and $m$. For details see e.g. [17]. Furthermore, if the group $G$ carries a bi-invariant metric, then the Riemannian covariant derivative $\frac{D}{d t}$ on $G$ coincides with ${ }^{0} \frac{D}{d t}[12$, Chapter $\mathrm{X}]$.
} 


\section{Hermite subdivision on manifolds via parallel transport}

Starting with a linear Hermite subdivision operator $S_{A}$ satisfying the spectral condition (2), we define a subdivision operator $U$ in a surface or a matrix group M.

Recall that we can write $S_{A}$ in the form

$$
S_{A}\left(\begin{array}{l}
p \\
v
\end{array}\right)_{i}=\sum_{j \in \mathbb{Z}}\left(\begin{array}{ll}
a_{i-2 j} & b_{i-2 j} \\
c_{i-2 j} & d_{i-2 j}
\end{array}\right)\left(\begin{array}{l}
p_{j} \\
v_{j}
\end{array}\right)=\left(\begin{array}{l}
\sum_{j \in \mathbb{Z}} a_{i-2 j} p_{j}+b_{i-2 j} v_{j} \\
\sum_{j \in \mathbb{Z}} c_{i-2 j} p_{j}+d_{i-2 j} v_{j}
\end{array}\right) .
$$

The reproduction of constants (3) is characterized by the conditions $\sum_{j \in \mathbb{Z}} a_{i-2 j}=1$ and $\sum_{j \in \mathbb{Z}} c_{i-2 j}=0$. This allows us to rewrite (11) as

$$
S_{A}\left(\begin{array}{c}
p \\
v
\end{array}\right)_{i}=\left(\begin{array}{c}
m_{i}+\sum_{j \in \mathbb{Z}} a_{i-2 j}\left(p_{j}-m_{i}\right)+b_{i-2 j} v_{j} \\
\sum_{j \in \mathbb{Z}} c_{i-2 j}\left(p_{j}-m_{i}\right)+d_{i-2 j} v_{j}
\end{array}\right),
$$

for any base point sequence $m$. We use (12) to define a subdivision operator $U$ that takes arguments in $\ell(T M)$.

Consider input data $\left(\begin{array}{l}p \\ v\end{array}\right) \in \ell(T M)$. For the base point sequence $m \in \ell(M)$ we either choose

$$
m_{i}=p_{i} \quad \text { or } \quad m_{i}=\mu_{p_{i}, p_{i+1}} \quad \text { for } i \in \mathbb{Z} .
$$

In [20] these base point sequences have been used for the $C^{1}$ and $C^{2}$ analysis of manifold-valued subdivision rules. It was shown in $[9,21]$, however, that base point sequences have to be chosen in a more sophisticated manner if one wants to obtain higher smoothness results.

Based on (12) we now define the subdivision operator $U$ for manifold-valued data:

$$
U\left(\begin{array}{l}
p \\
v
\end{array}\right)_{i}=\left(\begin{array}{c}
r_{i} \\
\mathrm{P}_{m_{i}}^{r_{i}}\left(w_{i}\right)
\end{array}\right)
$$

$$
\text { where }\left\{\begin{array}{l}
r_{i}=m_{i} \oplus \sum_{j \in \mathbb{Z}} a_{i-2 j}\left(p_{j} \ominus m_{i}\right)+b_{i-2 j} \mathrm{P}_{p_{j}}^{m_{i}}\left(v_{j}\right), \\
w_{i}=\sum_{j \in \mathbb{Z}} c_{i-2 j}\left(p_{j} \ominus m_{i}\right)+d_{i-2 j} \mathrm{P}_{p_{j}}^{m_{i}}\left(v_{j}\right)
\end{array}\right.
$$

In Section 6 we show that the successively generated data $\left(\begin{array}{l}p \\ v\end{array}\right), D^{-1} U\left(\begin{array}{l}p \\ v\end{array}\right)$, $D^{-2} U^{2}\left(\begin{array}{l}p \\ v\end{array}\right), \ldots$ converge to a curve and its derivative.

Note that if $M$ is a matrix group, then $U$ is invariant w.r.t. both left and right multiplication. Furthermore, if the linear operator $S_{A}$ is interpolatory, then obviously so is $U$.

We mention that $U$ can be defined analogously in the more general cases of Riemannian manifolds and Lie groups.

Example 2 Consider the matrix group $\mathrm{SO}(3)=\left\{p \in \mathbb{R}^{3 \times 3}: p\right.$ is orthogonal and $\operatorname{det}(p)>0\}$. The tangent space at $p \in \mathrm{SO}(3)$ is given by $T_{p} \mathrm{SO}(3)=\left\{v \in \mathbb{R}^{3 \times 3}\right.$ : $p^{-1} v$ is skew-symmetric $\}$. 

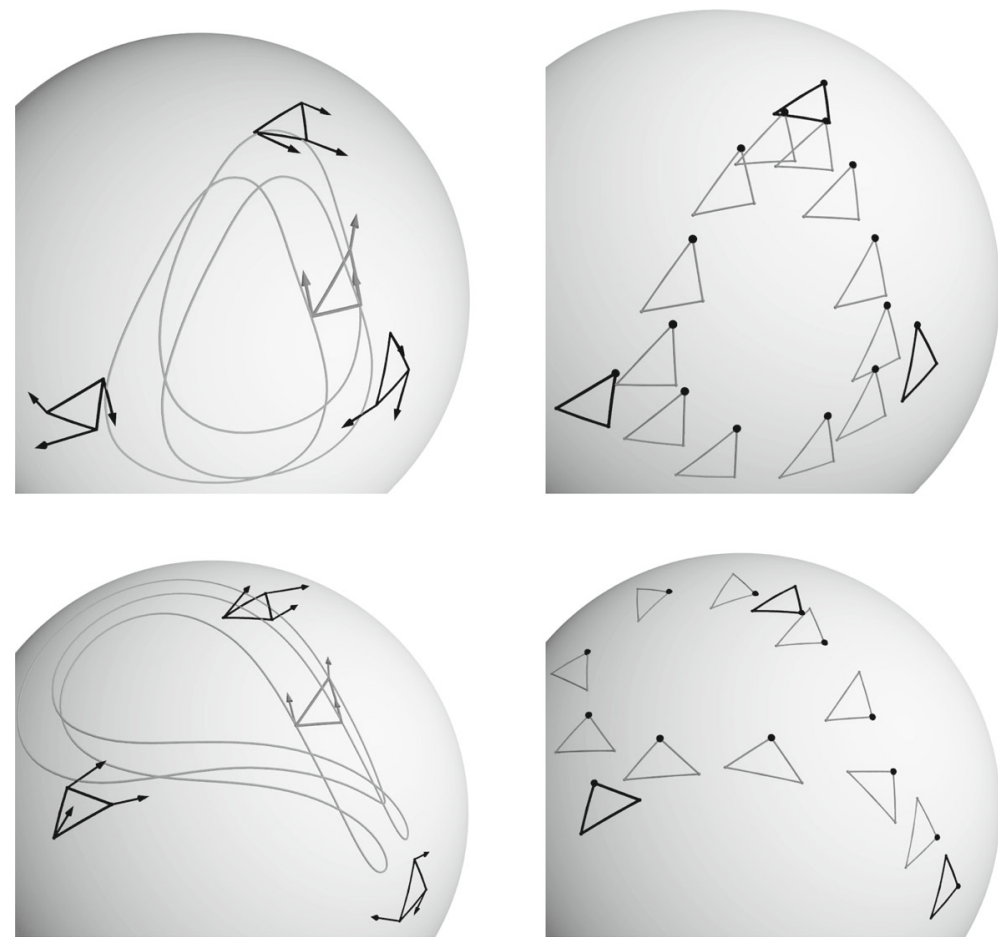

Fig. 2 The SO(3)-valued Hermite subdivision scheme of Example 2 with respect to the (0) parallel transport. Input data are represented by spherical triangles. Upper and lower left figures: Limit curves of point-vector input data and one triangle of the second iteration step. Upper and lower right figures: second iteration step (tangent vectors are omitted)

We consider the parallel transport version of the linear Hermite scheme introduced in Example 1, see Fig. 2. Recall from Section 3.3 that for $p, q \in \mathrm{SO}(3)$ and $v \in$ $T_{p} \mathrm{SO}(3)$ the operators $\oplus, \ominus$ are given by

$$
p \oplus v=p \exp \left(p^{-1} v\right) \quad \text { and } \quad q \ominus p=p \log \left(p^{-1} q\right),
$$

where exp is the matrix exponential and log is the matrix logarithm.

For input data $\left(\begin{array}{l}p \\ v\end{array}\right) \in \ell(T \mathrm{SO}(3))$ we choose the base point sequence $m$ as the midpoints of consecutive points of $p$ :

$$
m_{2 i}=m_{2 i+1}=\mu_{p_{i+1}, p_{i}}=p_{i+1} \oplus \frac{1}{2}\left(p_{i} \ominus p_{i+1}\right) .
$$

Furthermore, for $i, j \in \mathbb{Z}$ we introduce the following sequences:

$$
\begin{aligned}
& \mathbf{v}_{i, j}=p_{i} \ominus m_{j}, \\
& \mathbf{w}_{j, i}=\mathrm{P}_{p_{j}}^{m_{i}}\left(v_{j}\right)= \begin{cases}m_{i} p_{j}^{-1} v_{j} & \text { for the }(+) \text { parallel transport, } \\
v_{j} p_{j}^{-1} m_{i} & \text { for the (-) parallel transport, } \\
\mu_{p_{j}, m_{i}} p_{j}^{-1} v_{j} p_{j}^{-1} \mu_{p_{j}, m_{i}} & \text { for the (0) parallel transport. }\end{cases}
\end{aligned}
$$


The operator $U$ of (13) is given by

$$
U\left(\begin{array}{l}
p \\
v
\end{array}\right)_{i}=\left(\begin{array}{c}
r_{i} \\
\mathrm{P}_{m_{i}}^{r_{i}}\left(w_{i}\right)
\end{array}\right)
$$

where

$$
\begin{aligned}
r_{2 i} & =m_{2 i} \oplus \frac{1}{8}\left(\frac{48}{25} \mathbf{v}_{i+1,2 i}+\frac{152}{25} \mathbf{v}_{i, 2 i}-\frac{29}{25} \mathbf{w}_{i+1,2 i}+\frac{31}{25} \mathbf{w}_{i, 2 i}\right), \\
w_{2 i} & =\frac{1}{8}\left(\frac{29}{50} \mathbf{v}_{i+1,2 i}-\frac{29}{50} \mathbf{v}_{i, 2 i}+\frac{13}{20} \mathbf{w}_{i+1,2 i}+\frac{277}{100} \mathbf{w}_{i, 2 i}\right), \\
r_{2 i+1} & =m_{2 i+1} \oplus \frac{1}{8}\left(\frac{152}{25} \mathbf{v}_{i+1,2 i+1}+\frac{48}{25} \mathbf{v}_{i, 2 i+1}-\frac{31}{25} \mathbf{w}_{i+1,2 i+1}+\frac{29}{25} \mathbf{w}_{i, 2 i+1}\right), \\
w_{2 i+1} & =\frac{1}{8}\left(\frac{29}{50} \mathbf{v}_{i+1,2 i+1}-\frac{29}{50} \mathbf{v}_{i, 2 i+1}+\frac{277}{100} \mathbf{w}_{i+1,2 i+1}+\frac{13}{20} \mathbf{w}_{i, 2 i+1}\right) .
\end{aligned}
$$

The coefficients are taken from Example 1.

We consider the bi-invariant inner product $\langle u, v\rangle=\operatorname{trace}\left(u v^{T}\right)$ on $\mathrm{SO}(3)$. This bi-invariant inner product coincides with the standard inner product induced by $\mathbb{R}^{9}$, since trace $\left(u v^{T}\right)=\sum_{i, j} u_{i j} v_{i j}$. Therefore, $\mathrm{SO}(3)$ is a surface which carries a biinvariant inner product. It is known that the (0) parallel transport defined above coincides with the surface parallel transport (the same is true for the exponential mapping). Therefore, the above calculations are also valid if $\mathrm{SO}(3)$ is viewed as a surface.

\section{Proximity inequalities}

In order to conclude convergence and smoothness of ordinary manifold-valued subdivision rules, the proximity method was introduced, see [18, 19] and others. This method requires to establish inequalities on the difference between linear subdivision rules and manifold-valued subdivision rules. Since we need a variety of norms to state the proximity condition, we summarize all of them in the following section:

\subsection{Different types of norms}

The notation $\|v\|$, where $v$ is an element of $V=\mathbb{R}^{n}$, means that we use the Euclidean norm. On matrix groups we use the Frobenius norm $\|g\|^{2}=\operatorname{trace}\left(g g^{T}\right)$. As already mentioned in Example 2, the Frobenius norm corresponds to the Euclidean norm, if the matrix entries are put into a column vector. From this norm on $V$ we induce the Euclidean norm $\left\|\left(\begin{array}{c}v_{0} \\ v_{1}\end{array}\right)\right\|=\left(\left\|v_{0}\right\|^{2}+\left\|v_{1}\right\|^{2}\right)^{\frac{1}{2}}$ on $V^{2}$. On the space $L(V)^{2 \times 2}$ we use the operator norm

$$
\left\|\left(\begin{array}{ll}
a & b \\
c & d
\end{array}\right)\right\|=\sup \left\{\left\|\left(\begin{array}{ll}
a & b \\
c & d
\end{array}\right)\left(\begin{array}{l}
v_{0} \\
v_{1}
\end{array}\right)\right\|, \text { where }\left\|\left(\begin{array}{l}
v_{0} \\
v_{1}
\end{array}\right)\right\|=1\right\},
$$


where $\left(\begin{array}{ll}a & b \\ c & d\end{array}\right) \in L(V)^{2 \times 2}$ and $\left(\begin{array}{l}v_{0} \\ v_{1}\end{array}\right) \in V^{2}$. We equip the space of sequences $\ell\left(V^{2}\right)$ with the norm

$$
\left\|\left(\begin{array}{l}
p \\
v
\end{array}\right)\right\|_{\infty}=\sup _{i \in \mathbb{Z}}\left\|\left(\begin{array}{c}
p_{i} \\
v_{i}
\end{array}\right)\right\|
$$

and denote by $\ell^{\infty}\left(V^{2}\right)$ the space of all sequences which are bounded with respect to this norm. Similarly we define a norm for $A \in \ell\left(L(V)^{2 \times 2}\right)$ :

$$
\|A\|_{\infty}=\sup _{i \in \mathbb{Z}}\left\|A_{i}\right\|
$$

and denote by $\ell^{\infty}\left(L(V)^{2 \times 2}\right)$ the space of bounded sequences.

A linear subdivision operator $S_{A}$ as defined in Eq. 1 restricts to an operator $\ell^{\infty}\left(V^{2}\right) \rightarrow \ell^{\infty}\left(V^{2}\right)$. This follows from $\left\|S_{A}\left(\begin{array}{c}p \\ v\end{array}\right)\right\|_{\infty} \leq d\|A\|_{\infty}\left\|\left(\begin{array}{c}p \\ v\end{array}\right)\right\|_{\infty}$, where $d$ is a positive integer such that the support of $A$ is contained in $[-d, d]$. Therefore $S_{A}$ has an induced operator norm, which we denote by $\left\|S_{A}\right\|_{\infty}$.

We mention that for the proofs of the next section, the particular choices of the norms on $V$ and $V^{2}$ are not important. We will only need the Euclidean norm in Example 3. What we will use, however, are the following facts concerning the equivalence of norms: Since in every finite dimensional vector space, any two norms are equivalent, the Euclidean norm $\left\|\left(\begin{array}{c}v_{0} \\ v_{1}\end{array}\right)\right\|$ on $V^{2}$ is equivalent to $\left\|\left(\begin{array}{c}v_{0} \\ v_{1}\end{array}\right)\right\|^{\prime}=$ $\max \left\{\left\|v_{0}\right\|,\left\|v_{1}\right\|\right\}$. That is, there exist constants $c_{1}, c_{1}>0$ such that

$$
c_{1}\left\|\left(\begin{array}{c}
v_{0} \\
v_{1}
\end{array}\right)\right\|^{\prime} \leq\left\|\left(\begin{array}{c}
v_{0} \\
v_{1}
\end{array}\right)\right\| \leq c_{2}\left\|\left(\begin{array}{c}
v_{0} \\
v_{1}
\end{array}\right)\right\|^{\prime} .
$$

It follows immediately that also the norms $\left\|\left(\begin{array}{c}p \\ v\end{array}\right)\right\|_{\infty}^{\prime}=\sup _{i}\left\|\left(\begin{array}{c}p_{i} \\ v_{i}\end{array}\right)\right\|^{\prime}$ and $\left\|\left(\begin{array}{c}p \\ v\end{array}\right)\right\|_{\infty}$ on $\ell\left(V^{2}\right)$ are equivalent with the same constants:

$$
c_{1}\left\|\left(\begin{array}{c}
p \\
v
\end{array}\right)\right\|_{\infty}^{\prime} \leq\left\|\left(\begin{array}{c}
p \\
v
\end{array}\right)\right\|_{\infty} \leq c_{2}\left\|\left(\begin{array}{c}
p \\
v
\end{array}\right)\right\|_{\infty}^{\prime}
$$

\subsection{The proximity condition for Hermite schemes}

Consider a linear Hermite subdivision operator $S_{A}$ and a manifold-valued Hermite subdivision operator $U$. Then the proximity condition, introduced by [15] for Hermite schemes, is given by

$$
\left\|\left(U-S_{A}\right)\left(\begin{array}{c}
p \\
v
\end{array}\right)\right\|_{\infty} \leq c\left\|\left(\begin{array}{c}
\Delta p \\
v
\end{array}\right)\right\|_{\infty}^{2}
$$

Here $c$ is a constant and $\Delta$ denotes the forward difference operator $\Delta p_{i}=p_{i+1}-$ $p_{i}$ for $i \in \mathbb{Z}$.

To conclude $C^{1}$ convergence of $U$ from convergence of $S_{A}$, it is required that condition (15) is fulfilled whenever $\left\|\left(\begin{array}{c}p \\ v\end{array}\right)\right\|_{\infty}$ is bounded and $\left\|\left(\begin{array}{c}\Delta p \\ v\end{array}\right)\right\|_{\infty}$ is small enough.

In the following we prove that the proximity condition (15) holds between a linear operator $S_{A}$ and the $T M$-valued operator $U$ constructed from $S_{A}(13)$, where $M$ is a surface or matrix group. 
Recall from Eq. 13 that we defined sequences $r, w$ by

$$
\begin{aligned}
r_{i} & =m_{i} \oplus \sum_{j} a_{i-2 j}\left(p_{j} \ominus m_{i}\right)+b_{i-2 j} \mathrm{P}_{p_{j}}^{m_{i}}\left(v_{j}\right), \\
w_{i} & =\sum_{j} c_{i-2 j}\left(p_{j} \ominus m_{i}\right)+d_{i-2 j} \mathrm{P}_{p_{j}}^{m_{i}}\left(v_{j}\right),
\end{aligned}
$$

for $i \in \mathbb{Z}$. We also define $r^{\text {lin }}$ and $w^{\text {lin }}$, which are the linear versions of $r$ and $w$. This means that $\oplus$ and $\ominus$ are replaced by + and - respectively and $\mathrm{P}_{p_{j}}^{m_{i}}\left(v_{j}\right)$ is replaced by $v_{j}$. Therefore, in order to prove (15), we have to show the inequalities:

$$
\begin{aligned}
\left\|r-r^{\operatorname{lin}}\right\|_{\infty} & \leq c\left\|\left(\begin{array}{c}
\Delta p \\
v
\end{array}\right)\right\|_{\infty}^{2}, \\
\left\|\mathrm{P}_{m}^{r}(w)-w^{\operatorname{lin}}\right\|_{\infty} & \leq c\left\|\left(\begin{array}{c}
\Delta p \\
v
\end{array}\right)\right\|_{\infty}^{2} .
\end{aligned}
$$

The main ingredient in the proof is the following lemma:

Lemma 1 Let $M$ be a surface or matrix group. Then for $p, m \in M$ and tangent vectors $v$ the following linearizations hold:

$$
\begin{aligned}
& p \oplus v=p+v+O\left(\|v\|^{2}\right) \quad \text { as } \quad v \rightarrow 0, \\
& m \ominus p=m-p+O\left(\|m-p\|^{2}\right) \quad \text { as } \quad m \rightarrow p, \\
& \mathrm{P}_{m}^{p}(v)=v+O(\|m-p\|\|v\|) \quad \text { as } \quad m \rightarrow p .
\end{aligned}
$$

In the case that $M$ is a surface, $\mathrm{P}_{m}^{p}$ denotes the parallel transport along the geodesic connecting $p$ and $m$. If $M$ is a matrix group, then $\mathrm{P}_{m}^{p}$ denotes one of the $(+),(-)$, or $(0)$ parallel transports.

Proof In a chart of $M$, (19) and (20) are exactly the well-known linearization of the exponential map. In order to prove (21), we first observe that $(m, v) \mapsto \mathrm{P}_{m}^{p}(v)$ is smooth. On a surface, this can be deduced from the fact that the solution of an ordinary differential equation depends smoothly on the initial data. In the matrix group case, the smoothness of this map follows from the definition of the parallel transport. Restricting to a unit vector $v$ and using Taylor expansion in a chart at $m=p$, we obtain

$$
\mathrm{P}_{m}^{p}(v)=\mathrm{P}_{p}^{p}(v)+O(\|m-p\|)=v+O(\|m-p\|) \quad \text { as } \quad m \rightarrow p, v=\text { const. }
$$

Since $\mathrm{P}_{m}^{p}$ is a linear map, for a general $v$, we obtain $\mathrm{P}_{m}^{p}(v)=v+O(\|m-p\|\|v\|)$ as $m \rightarrow p$. This completes the proof.

Corollary 1 (Proximity inequalities) Let $M$ be a surface or matrix group. Consider bounded input data $\left(\begin{array}{l}p \\ v\end{array}\right)$ on TM and a base point sequence $m$, which is either given 
by $m_{i}=p_{i}$ or $m_{i}=\mu_{p_{i}, p_{i+1}}$ for $i \in \mathbb{Z}$. Then the sequences $r$ and $w$ as defined in (16) satisfy

$$
\begin{aligned}
r_{i} & =r_{i}^{\operatorname{lin}}+O\left(\sup _{j}\left\|m_{i}-p_{j}\right\|^{2}\right)+O\left(\sup _{j}\left\|m_{i}-p_{j}\right\| \sup _{j}\left\|v_{j}\right\|\right)+O\left(\sup _{j}\left\|v_{j}\right\|^{2}\right), \\
w_{i} & =w_{i}^{\operatorname{lin}}+O\left(\sup _{j}\left\|m_{i}-p_{j}\right\| \sup _{j}\left\|v_{j}\right\|\right), \\
\mathrm{P}_{m_{i}}^{r_{i}}\left(w_{i}\right) & =w_{i}^{\operatorname{lin}}+O\left(\sup _{j}\left\|m_{i}-p_{j}\right\|^{2}\right)+O\left(\sup _{j}\left\|m_{i}-p_{j}\right\| \sup _{j}\left\|v_{j}\right\|\right)+O\left(\sup _{j}\left\|v_{j}\right\|^{2}\right),
\end{aligned}
$$

for $m \rightarrow p$ and $v \rightarrow 0$ and $i \in \mathbb{Z}$. In particular, the proximity inequalities (17) and (18) follow.

Proof Using Lemma 1, the results for $r$ and $w$ immediately follow. Similarly, we can show that $\left\|r_{i}-m_{i}\right\|=O\left(\sup _{j}\left\|p_{j}-m_{i}\right\|\right)+O\left(\sup _{j}\left\|v_{j}\right\|\right)$. This implies

$$
\begin{aligned}
\mathrm{P}_{m_{i}}^{r_{i}}\left(w_{i}\right)= & w_{i}+O\left(\left\|r_{i}-m_{i}\right\|\left\|w_{i}\right\|\right) \\
= & w_{i}^{\operatorname{lin}}+O\left(\sup _{j}\left\|m_{i}-p_{j}\right\| \sup _{j}\left\|v_{j}\right\|\right)+O\left(\left\|r_{i}-m_{i}\right\|\left\|w_{i}\right\|\right) \\
= & w_{i}^{\operatorname{lin}}+O\left(\sup _{j}\left\|m_{i}-p_{j}\right\|^{2}\right)+O\left(\sup _{j}\left\|m_{i}-p_{j}\right\| \sup _{j}\left\|v_{j}\right\|\right) \\
& +O\left(\sup _{j}\left\|v_{j}\right\|^{2}\right),
\end{aligned}
$$

Furthermore, Lemma 1 implies $\sup _{j}\left\|m_{i}-p_{j}\right\| \leq c\|\Delta p\|_{\infty}$. Thus the above equations show that $\left\|r-r^{\operatorname{lin}}\right\|_{\infty} \leq c \max \left\{\|\Delta p\|_{\infty}^{2},\|v\|_{\infty}^{2}\right\}$ and $\left\|\mathrm{P}_{m}^{r}(w)-w^{\operatorname{lin}}\right\|_{\infty} \leq$ $c \max \left\{\|\Delta p\|_{\infty}^{2},\|v\|_{\infty}^{2}\right\}$. By the equivalence of norms (14), the proximity inequality (17) and (18) are proved. This completes the proof.

\section{Results}

In the previous section we have gathered all proximity inequalities we need to prove $C^{1}$ convergence of the manifold-valued Hermite scheme defined in Section 4. Our main theorem (Theorem 2) is analogous to Theorem 27 of [15].

Before we state the theorem, we have to introduce the Taylor operator. In linear Hermite subdivision, the Taylor operator is the natural analogue to the forward difference operator $\Delta p_{i}=p_{i+1}-p_{i}$ for $i \in \mathbb{Z}$, see [14]. It acts on $\ell\left(V^{2}\right)$ and is defined by

$$
T=\left(\begin{array}{cc}
\Delta & -1 \\
0 & \Delta
\end{array}\right) .
$$

In [14] this operator is called complete Taylor operator. We have the following result:

Theorem 1 (Merrien and Sauer, 2012) Let $S_{A}$ be a linear subdivision operator which satisfies the spectral condition (2). Then we have the following 
1. There exists a linear subdivision operator $S_{B}$ such that

$$
2 T S_{A}=S_{B} T \text {. }
$$

We call $S_{B}$ the Taylor scheme of $S_{A}$.

2. If there exists $N \in \mathbb{N}$ such that $\left\|S_{B}^{N}\right\|_{\infty}<1$, then the linear Hermite scheme associated to $S_{A}$ is $C^{1}$ convergent.

Now we can state the main result of our paper:

Theorem 2 Let $S_{A}$ be a linear subdivision operator whose mask A satisfies the spectral condition (2), and let $S_{B}$ be the Taylor scheme of $S_{A}$ (Theorem 1). Let $M$ be a surface or a matrix group and let $U$ be the manifold-valued analogue of $S_{A}$ given by (13). Then we have the following result:

If there exists $N \in \mathbb{N}$ such that $\left\|S_{B}^{N}\right\|_{\infty}<1$, then the Hermite scheme $\left(\begin{array}{c}p \\ v\end{array}\right)$, $D^{-1} U\left(\begin{array}{l}p \\ v\end{array}\right), D^{-2} U^{2}\left(\begin{array}{l}p \\ v\end{array}\right), \ldots$ is $C^{1}$ convergent whenever $\left(\begin{array}{l}p \\ v\end{array}\right)$ are dense enough.

The statement of the theorem remains true if "surface" is replaced by "Riemannian manifold" and "matrix group" by "Lie group".

Proof It is proved in [15] that $\left\|S_{A}^{N}\right\|_{\infty}<1$ for some integer $N$ together with the proximity condition implies $C^{1}$ convergence of the manifold-valued Hermite scheme. Therefore, the result follows from Section 5 and [15].

Note that the input data does not have to be bounded. This follows from the fact that on any compact interval the limit curve only depends on finitely many points of the input data. We can therefore w.l.o.g. assume that $\left\|\left(\begin{array}{c}p \\ v\end{array}\right)\right\|_{\infty}$ is bounded.

The global embedding theorem states that any Riemannian manifold can be isometrically embedded as a surface into a Euclidean space of sufficiently high dimension. The smoothness is preserved by this embedding. Our result applies to this surface. Furthermore, by Ado's theorem, any Lie group is locally isomorphic to a matrix group. Therefore, the generalized statement is also true. This completes the proof.

Remark 1 We would like to remark on possible generalizations of this result, which are topics of future research.

It would be natural to consider schemes which produce more than one derivative, i.e. schemes refining sequences with more than two components, with the $k$ th component representing the $(k-1)$ st derivative. This has been studied in the linear case, see e.g. [14]. The manifold version of this are data sampled from the jet bundle.

Furthermore, one can study Hermite subdivision schemes which produce limit functions of higher regularity than the minimal one (that is, the number of derivatives). In the linear case, examples of such Hermite schemes can be found in e.g. [2, 10]. It would be interesting to show that the limit functions of the manifold-valued analogue constructed in Section 4 inherit regularity higher than $C^{1}$ from their linear counterparts.

We believe that such generalizations become quite technical: Available results from point-subdivision in manifolds suggest that $C^{2}$ smoothness can be achieved in 
general, but higher smoothness requires care in defining the manifold analogue of a linear scheme $[9,21]$.

Example 3 We consider the linear subdivision operator $S_{A}$ whose mask is defined in Example 1. In [14] it is shown that the operator $S_{B}$ satisfying $2 T S_{A}=S_{B} T$ has the mask

$$
B_{-1}=\frac{1}{4}\left(\begin{array}{rr}
\frac{48}{25} & -\frac{29}{25} \\
\frac{29}{50} & \frac{13}{20}
\end{array}\right), \quad B_{0}=\frac{1}{4}\left(\begin{array}{rr}
\frac{179}{50} & -\frac{73}{100} \\
0 & \frac{53}{25}
\end{array}\right), \quad B_{1}=\frac{1}{4}\left(\begin{array}{rr}
\frac{67}{50} & \frac{47}{100} \\
-\frac{29}{50} & \frac{123}{100}
\end{array}\right) .
$$

We prove $\left\|S_{B}\right\|_{\infty}<1$. The norm of a subdivision operator is given by

$$
\left\|S_{B}\right\|_{\infty}=\sup \left\{\left\|S_{B}\left(\begin{array}{c}
p \\
v
\end{array}\right)\right\|_{\infty}:\left\|\left(\begin{array}{c}
p \\
v
\end{array}\right)\right\|_{\infty}=1\right\} .
$$

It is well known that

$$
\left\|S_{B}\right\|_{\infty}=\max \left\{\sum_{j \in \mathbb{Z}}\left\|B_{-2 j}\right\|, \sum_{j \in \mathbb{Z}}\left\|B_{-2 j+1}\right\|\right\} .
$$

Therefore, we have to prove that $\max \left\{\left\|B_{0}\right\|,\left\|B_{-1}\right\|+\left\|B_{1}\right\|\right\}<1$. The operator norm of a matrix w.r.t. to the Euclidean norm equals the spectral norm, therefore

$$
\left\|B_{i}\right\|=\sqrt{\lambda_{\max }\left(B_{i}^{T} B_{i}\right)},
$$

where $\lambda_{\max }$ is the largest eigenvalue of the matrix $B_{i}^{T} B_{i}$ for $i=-1,0,1$. This yields

$$
\begin{aligned}
\lambda_{\max }(0) & =\frac{178437+73 \sqrt{1651145}}{320000}<1, \\
\lambda_{\max }(-1) & =\frac{57909+5 \sqrt{75106529}}{320000}<\frac{36}{100}, \\
\lambda_{\max }(1) & =\frac{19329+11 \sqrt{38537}}{160000}<\frac{16}{100} .
\end{aligned}
$$

This implies that $\left\|S_{B}\right\|_{\infty}<1$ and therefore the $C^{1}$ convergence of the linear Hermite scheme defined by $S_{A}$. Furthermore, Theorem 2 shows that its parallel transport version on any Riemannian manifold or Lie group is $C^{1}$ convergent for dense enough input data. In particular this includes $\mathrm{SO}(3)$, i.e., our Example 2.

\subsection{Conclusion}

We have studied a manifold-valued analogue of linear Hermite subdivision schemes which is defined by using the parallel transport operator of the manifold. This construction is intrinsic and gives rise to a $C^{1}$ convergent nonlinear subdivision scheme, if the input data are dense enough and the Taylor scheme is appropriately bounded (Theorem 2). Similar to most convergence and smoothness results of subdivision rules in general manifolds, the main ingredient of the proof is the method of proximity. 
Acknowledgments Open access funding provided by Graz University of Technology. The author would like to thank Johannes Wallner for helpful discussions on earlier versions of this paper and gratefully acknowledges the suggestions of the anonymous reviewers.

Open Access This article is distributed under the terms of the Creative Commons Attribution 4.0 International License (http://creativecommons.org/licenses/by/4.0/), which permits unrestricted use, distribution, and reproduction in any medium, provided you give appropriate credit to the original author(s) and the source, provide a link to the Creative Commons license, and indicate if changes were made.

\section{References}

1. do Carmo, M.P.: Riemannian Geometry. Birkhäuser Verlag (1992)

2. Conti, C., Merrien, J.L., Romani, L.: Dual Hermite subdivision schemes of de Rham-type. BIT Numer. Math. 54, 955-977 (2014)

3. Dubuc, S.: Scalar and Hermite subdivision schemes. Appl. Comput. Harmon. Anal. 21(3), 376-394 (2006)

4. Dubuc, S., Merrien, J.L.: Convergent vector and Hermite subdivision schemes. Constr. Approx. 23(1), $1-22(2005)$

5. Dubuc, S., Merrien, J.L.: Approximation Theory XII. In: Neamtu, M., Schumaker, L.L. (eds.): De Rham transform of a Hermite subdivision scheme, pp. 121-132. Nashboro Press, Nashville, TN (2008)

6. Dubuc, S., Merrien, J.L.: Hermite subdivision schemes and Taylor polynomials. Constr. Approx. 29(2), 219-245 (2009)

7. Dyn, N., Levin, D.: Approximation Theory VIII. Vol 2: Wavelets and Multilevel Approximation. In: Chui, C.K., Schumaker, L.L. (eds.): Analysis of Hermite-type subdivision schemes, pp. 117-124. World Sci., River Edge, NJ (1995)

8. Dyn, N., Levin, D.: Spline Functions and the Theory of Wavelets. In: Dubuc, S., Deslauriers, G. (eds.): Analysis of Hermite-interpolatory subdivision schemes, pp. 105-113. Amer. Math. Soc., Providence, RI (1999)

9. Grohs, P.: A general proximity analysis of nonlinear subdivision schemes. SIAM J. Math. Anal. 42(2), 729-750 (2010)

10. Han, B., Yu, T., Xue, Y.: Noninterpolatory Hermite subdivision schemes. Math. Comput. 74(251), 1345-1367 (2005)

11. Helgason, S.: Differential Geometry, Lie Groups, and Symmetric Spaces. Academic Press (1979)

12. Kobayashi, S., Nomizu, K.: Foundations of differential geometry, vol. 2. Wiley (1969)

13. Merrien, J.L.: A family of Hermite interpolants by bisection algorithms. Numer. Algorithms 2(2), 187-200 (1992)

14. Merrien, J.L., Sauer, T.: From Hermite to stationary subdivision schemes in one and several variables. Adv. Comput. Math. 36(4), 547-579 (2012)

15. Moosmüller, C.: $C^{1}$ analysis of Hermite subdivision schemes on manifolds. SIAM J. Numer. Anal. 54(5), 3003-3031 (2016)

16. Onishchik, A.L., Vinberg, E.B.: Lie Groups and Lie Algebras I: Foundations of Lie Theory. Springer (1993)

17. Postnikov, M.M.: Geometry VI: Riemannian Geometry. Springer (2001)

18. Wallner, J.: Smoothness analysis of subdivision schemes by proximity. Constr. Approx. 24, 289-318 (2006)

19. Wallner, J., Dyn, N.: Convergence and $C^{1}$ analysis of subdivision schemes on manifolds by proximity. Comput. Aided Geom. Des. 22(7), 593-622 (2005)

20. Wallner, J., Nava Yazdani, E., Weinmann, A.: Convergence and smoothness analysis of subdivision rules in Riemannian and symmetric spaces. Adv. Comput. Math. 34(2), 201-218 (2011)

21. Xie, G., Yu, T.: Smoothness equivalence properties of general manifold-valued data subdivision schemes. SIAM Journal on Multiscale Modeling \& Simulation 7(3), 1073-1100 (2009) 